\title{
Torque on a Rotating Ellipsoid in a Rarefied Gas
}

\author{
J. Halbritter \\ Institut für Theoretische Physik der Universität Erlangen-Nürnberg, Erlangen \\ (Z. Naturforsch. 29a, 1717-1722 [1974] ; received September 4, 1974)
}

\begin{abstract}
A particle, shaped as an ellipsoid of revolution and rotating about an arbitrary axis, ex. periences a torque in a rarefied monatomic gas because of the impact with the gas atoms. This torque is calculated for the case where the linear dimensions of the ellipsoid are small compared with the mean free path of the gas. The interaction of the gas atoms with the ellipsoidal surface is taken into account by means of the boundary condition for the distribution function in terms of an accommodation coefficient (diffuse and specular reflection). The torque is considered for prolate and oblate ellipsoids. The formulae obtained are of interest in the theory of the Brownian motion of a rotating particle in a rarefied gas.
\end{abstract}

It is well known that a small sphere moving with velocity $\boldsymbol{v}$ through a rarefied gas experiences a drag $\boldsymbol{K}$ which for low velocity (as compared with the veiocity of sound) is given by the formula of Epstein ${ }^{1}$. In the opposite case (radius of the sphere rarge compared with the mean free path) the usual Stokes formula is valid. A similar situation is found if one considers the torque on a rotating ellipsoid. In the hydrodynamical regime, the torque on rotating ellipsoids has been calculated by Gans ${ }^{2}$ (cf. also Reference $^{3}$ ). In the present paper the torque on a rotating ellipsoid of revolution is calculated in the limit where the linear dimension of the ellipsoid $L$ is small compared with the mean free path. The two cases, prolate and oblate ellipsoids, will be discussed separately. The calculations are limited to the case where $\Omega L$ is small compared with the velocity of sound $(\Omega$ is the angular velocity of the rotating ellipsoid). This is actually no serious restriction, since in the case of interest (the Brownian motion of a rotating particle) this condition is always fulfilled. The calculations of the torque on a rotating sphere or for a disk (rotating about an axis perpendicular to the surface) can even be done without this restriction.

The formulae for the torque obtained below are of interest in the study of the Brownian motion of a rotating particle in a rarefied gas. The torque is equal to the rate of change of the angular momentum of the rotating particle due to the interaction with the gas atoms. This interaction must therefore be specified. For the following calculations it is assumed that a fraction $(1-\alpha)$ of the atoms incident on the ellipsoid is specularly reflected, whereas the other atoms are re-emitted by the surface with a Maxwell distribution corresponding to the temperature of the ellipsoid (which is assumed to be equal to the temperature of the gas) and its motion. In the case considered, the presence of the ellipsoid does not alter the distribution of the atoms incident on its surface, which is a Maxwell distribution at rest.

\section{$\S 1$. General Formula for the Torque}

The force $\mathrm{d} \boldsymbol{K}$ on a surface element $\mathrm{d} S$ at rest with outer normal $\boldsymbol{n}$ pointing into the gas is given by

$$
\mathrm{d} \boldsymbol{K}=-\mathrm{d} S\left[\int_{-} m \boldsymbol{c}^{\prime}\left(\boldsymbol{c}^{\prime} \cdot \boldsymbol{n}\right) f_{-}\left(\boldsymbol{c}^{\prime}\right) \mathrm{d}^{3} c^{\prime}+\int_{+} m \boldsymbol{c}^{\prime}\left(\boldsymbol{c}^{\prime} \cdot \boldsymbol{n}\right) f_{+}\left(\boldsymbol{c}^{\prime}\right) \mathrm{d}^{3} c^{\prime}\right]
$$

The subscripts \pm on the integrals mean integration over $\boldsymbol{c}^{\prime} \cdot \boldsymbol{n}>0$ and $\boldsymbol{c}^{\prime} \cdot \boldsymbol{n}<0$. The distribution function of the gas atoms with mass $m$ incident on the surface element $\mathrm{d} S$ is denoted by $f_{-}$, whereas $f_{+}$denotes the distribution function of the gas atoms which leave the surface element. The functions $f_{+}$and $f_{-}$are connected by the boundary condition which will be stated below. Equation (1) is valid in a coordinate system where the surface element $\mathrm{d} S$ is at rest. Therefore one has to introduce a coordinate system attached to the body and rotating with angular velocity $\Omega$ with respect to the laboratory system. The velocity $\boldsymbol{c}^{\prime}$

Reprint requests to Dr. J. Halbritter, Institut für Theoretische Physik der Universität Erlangen-Nürnberg, D-8520 Erlangen, Glückstraße 6. 
of a gas atom in Eq. (1) is measured with respect to this rotating system. At every point $\boldsymbol{x}$ of the surface of the body, the velocity $\boldsymbol{c}^{\prime}$ is connected with the velocity $\boldsymbol{c}$ in the laboratory system by

$$
\boldsymbol{c}^{\prime}=\boldsymbol{c}-\boldsymbol{\Omega} \times \boldsymbol{x} .
$$

The distribution function of the gas atoms incident on a surface element $\mathrm{d} S$ at the point $\boldsymbol{x}$ is a Maxwell distribution in the rotating system with mean velocity $-\boldsymbol{\Omega} \times \boldsymbol{x}$ :

$$
f_{-}\left(\boldsymbol{c}^{\prime}\right)=n\left(m / 2 \pi k_{\mathrm{B}} T\right)^{3 / 2} \exp \left\{-\frac{m}{2 k_{\mathrm{B}} T}\left(\boldsymbol{c}^{\prime}+\boldsymbol{\Omega} \times \boldsymbol{x}\right)^{2}\right\} .
$$

Here $n$ is the number density, $T$ the temperature of the gas and $k_{\mathrm{B}}$ Boltzmann's constant.

In order to calculate the force $\mathrm{d} \boldsymbol{K}$, the connection between $f_{+}$and $f_{-}$must be given. The boundary condition in the rotating system will be written for $\boldsymbol{c}^{\prime} \cdot \boldsymbol{n}>0$ as

$$
\begin{aligned}
& \boldsymbol{c}^{\prime} \cdot \boldsymbol{n} f_{+}\left(\boldsymbol{c}^{\prime}\right)=(1-\alpha) \boldsymbol{c}^{\prime} \cdot \boldsymbol{n} f_{-}\left(\boldsymbol{c}^{\prime}-2 \boldsymbol{c}^{\prime} \cdot \boldsymbol{n} \boldsymbol{n}\right) \\
&+\alpha \boldsymbol{c}^{\prime} \cdot \boldsymbol{n} \exp \left\{-\frac{m c^{\prime 2}}{2 k_{\mathrm{B}} T}\right\}\left[2 \pi\left(\frac{k_{\mathrm{B}} T}{m}\right)^{2}\right]^{-1} \int(-\boldsymbol{c} \cdot \boldsymbol{n}) f_{-}(\boldsymbol{c}) \mathrm{d}^{3} c .
\end{aligned}
$$

The velocity $\tilde{\boldsymbol{c}}=\boldsymbol{c}^{\prime}-2\left(\boldsymbol{c}^{\prime} \cdot \boldsymbol{n}\right) \boldsymbol{n}$ in the distribution function of the incident atoms represents the velocity of an atom before collision with the surface element $\mathrm{d} S$ which after specular reflection is changed into $\boldsymbol{c}^{\prime}$. The accommodation coefficient is denoted by $\alpha$. By substituting Eq. (4) into Eq. (1) one obtains

$$
\begin{aligned}
\mathrm{d} \boldsymbol{K}=-\mathrm{d} S\left[\alpha \int_{-} m \boldsymbol{c}^{\prime}\left(\boldsymbol{c}^{\prime} \cdot \boldsymbol{n}\right) f_{-}\left(\boldsymbol{c}^{\prime}\right) \mathrm{d}^{3} c^{\prime}\right. & +2(1-\alpha) \boldsymbol{n} \int_{-} m\left(\boldsymbol{c}^{\prime} \cdot \boldsymbol{n}\right)^{2} f_{-}\left(\boldsymbol{c}^{\prime}\right) \mathrm{d}^{3} c^{\prime} \\
& \left.+\boldsymbol{n} \frac{\pi}{4} \alpha \bar{c} \int_{-} m\left(-\boldsymbol{c}^{\prime} \cdot \boldsymbol{n}\right) f_{-}\left(\boldsymbol{c}^{\prime}\right) \mathrm{d}^{3} c^{\prime}\right] .
\end{aligned}
$$

In Eq. (5) a mean thermal velocity

$$
\bar{c}=\left(8 k_{\mathrm{B}} T / \pi m\right)^{1 / 2}
$$

has been introduced. In the following, the incident distribution function of Eq. (3) is approximated as

$$
f_{-}\left(\boldsymbol{c}^{\prime}\right) \approx n\left(m / 2 \pi k_{\mathrm{B}} T\right)^{3 / 2} \exp \left\{-\frac{m c^{\prime 2}}{2 k_{\mathrm{B}} T}\right\}\left[1-\frac{m}{k_{\mathrm{B}} T} \boldsymbol{c}^{\prime} \cdot(\boldsymbol{\Omega} \times \boldsymbol{x})\right] .
$$

This is justified if $\Omega L \ll \bar{c}$, where $L$ is of the order of the linear dimension of the rotating body. For a rotating Brownian particle $\Omega L / \bar{c} \sim(m / M)^{1 / 2}$, where $M$ is the mass of the rotating particle. This ratio is indeed very small. Equation (7) is now substituted into Equation (5). The Maxwell integrals can easily be performed. One obtains the following expression for the force $\mathrm{d} \boldsymbol{K}$ on the surface element $\mathrm{d} S$

$$
\mathrm{d} \boldsymbol{K}=-\mathrm{d} S \text { p } \boldsymbol{n}-\mathrm{d} S \varrho \bar{c}\left[\frac{\alpha}{4} \boldsymbol{\Omega} \times \boldsymbol{x}+\left(1-\frac{6-\pi}{8} \alpha\right) \boldsymbol{n} \boldsymbol{n} \cdot(\boldsymbol{\Omega} \times \boldsymbol{x})\right],
$$

where the mass density $\varrho=n m$ and the pressure $p=\left(k_{\mathrm{B}} T / m\right) \varrho$ have been introduced. The torque on a surface element $\mathrm{d} S$ is given by $\mathrm{d} \boldsymbol{M}=\boldsymbol{x} \times \mathrm{d} \boldsymbol{K}$. On integration over the surface the contribution of the first term on the right of Eq. (8) of course vanishes. The total torque $\boldsymbol{M}$ on the body is

$$
\boldsymbol{M}=-\varrho \bar{c}\left[\frac{\alpha}{4} \int \boldsymbol{x} \times(\Omega \times \boldsymbol{x}) \mathrm{d} S+\left(1-\frac{6-\pi}{8} \alpha\right) \int(\boldsymbol{x} \times \boldsymbol{n}) \Omega \cdot(\boldsymbol{x} \times \boldsymbol{n}) \mathrm{d} S\right] .
$$

This equation gives the torque on a rotating body of arbitrary shape. The integrals in Eq. (9) give different results for prolate and oblate ellipsoids of revolution. They are calculated in the appendix of this paper. In the following chapter the torque will be discussed separately for this two types of ellipsoids.
For a sphere and for a disk rotating about an axis perpendicular to the surface, the expansion of the incident distribution function as in Eq. (7) is not needed. One merely has to introduce the velocity $\boldsymbol{c}=\boldsymbol{c}^{\prime}+\boldsymbol{\Omega} \times \boldsymbol{x}$ instead of $\boldsymbol{c}^{\prime}$ as a new integration variable in the integrals occurring in Equation (5). 
Since $\boldsymbol{n} \cdot(\boldsymbol{\Omega} \times \boldsymbol{x})=0$ for the sphere and for the disk, the limits of integration are not changed $(\boldsymbol{c} \cdot \boldsymbol{n}=$ $\left.\boldsymbol{c}^{\prime} \cdot \boldsymbol{n}\right)$. Thus the torque on a sphere (radius $a$ ) rotating with angular velocity $\Omega$ can exactly be calculated from Equations (3), (5). The result is

$$
\boldsymbol{M}=-\alpha \frac{2 \pi}{3} \varrho \bar{c} a^{4} \Omega \text {. }
$$

The corresponding formula for a rotating disk with radius $a$ is

$$
\boldsymbol{M}=-\frac{1}{4} \alpha \varrho \bar{c} \pi a^{4} \Omega .
$$

It should be noted that the result of Epstein ${ }^{1,4}$ for the force $\boldsymbol{K}$ on a sphere moving with velocity $\boldsymbol{v}$ can also be obtained from Eq. (8) by the substitution $\boldsymbol{\Omega} \times \boldsymbol{x} \rightarrow \boldsymbol{v}:$

$$
\boldsymbol{K}=-\left(1+\frac{\pi}{8} \alpha\right) \frac{4 \pi}{3} \varrho \bar{c} a^{2} \boldsymbol{v} .
$$

The force on moving ellipsoids could also be obtained by this method.

\section{$\S 2$. Special Cases: Prolate and Oblate Ellipsoids of Revolution}

\section{a) Prolate Ellipsoids}

The symmetry axis (figure axis) of the ellipsoid is characterized by the unit vector $\boldsymbol{u}$. The major and minor semiaxes will be denoted by $a$ and $b(a>b)$; the eccentricity is given by $\varepsilon=\left(1-b^{2} / a^{2}\right)^{1 / 2}$. The two integrals oocurring in Eq. (9) are calculated in the appendix. The following result for the torque is obtained:

$$
\boldsymbol{M}=-\gamma_{\|} \boldsymbol{u} \boldsymbol{u} \cdot \boldsymbol{\Omega}-\gamma_{\perp}(\boldsymbol{\Omega}-\boldsymbol{u} \boldsymbol{u} \cdot \boldsymbol{\Omega}) .
$$

The two positive coefficients $\gamma_{\|}$and $\gamma_{\perp}$ determine the torque when the angular velocity $\Omega$ of the rotating ellipsoid is parallel or perpendicular to the symmetry axis $\boldsymbol{u}$. They are given by the following expressions:

$$
\begin{gathered}
\gamma_{\|}=\alpha \gamma_{\mathrm{s}}\left(1-\varepsilon^{2}\right)^{3 / 2} f_{2}(\varepsilon), \\
\gamma_{\perp}=\frac{1}{2} \gamma_{\mathrm{s}}\left(1-\varepsilon^{2}\right)^{1 / 2}\left[\alpha\left(f_{1}(\varepsilon)+\left(1-\varepsilon^{2}\right) f_{2}(\varepsilon)\right)\right. \\
\left.+3\left(1-\frac{6-\pi}{8} \alpha\right) \varepsilon^{4} f_{3}(\varepsilon)\right] .
\end{gathered}
$$

The functions $f_{1}, f_{2}$ and $f_{3}$ are given in the appendix. The coefficient

$$
\gamma_{\mathrm{s}}=\frac{2 \pi}{3} \varrho \bar{c} a^{4}
$$

determines according to Eq. (10) the torque on a sphere with radius $a: \boldsymbol{M}=-\alpha \gamma_{\mathrm{s}} \Omega$. For $\varepsilon=0$ the two coefficients $\gamma_{\|}$and $\gamma_{\perp}$ are equal to $\alpha \gamma_{\mathrm{s}}$ as it should be, whereas for $\varepsilon \rightarrow 1$ they tend to zero. The relaxation frequencies for the angular velocity $\Omega$ are given by $\omega_{\|}=\gamma_{\|} / \Theta_{\|}$and $\omega_{\perp}=\gamma_{\perp} / \Theta_{\perp}$, where $\Theta_{\|}$ and $\Theta_{\perp}$ are the corresponding moments of inertia for rotations parallel and perpendicular to the axis $\boldsymbol{u}$. The frequencies $\omega_{\|}$and $\omega_{\perp}$ are of the same order of magnitude. Their ratio $\chi=\alpha_{\|} / \alpha_{\perp}$ is given by

$$
\begin{aligned}
\chi=\alpha\left(2-\varepsilon^{2}\right) f_{2}\left[\alpha\left(f_{1}+\left(1-\varepsilon^{2}\right) f_{2}\right)\right. \\
\left.+3\left(1-\frac{6-\pi}{8} \alpha\right) \varepsilon^{4} f_{3}\right]^{-1} .
\end{aligned}
$$

For $\varepsilon$ in the range $0 \leqq \varepsilon \leqq 1$, the inequality

$$
1 \geqq \chi \geqq 3 \alpha\left[3 \alpha+4(1-\alpha)+\frac{\pi}{2} \alpha\right]^{-1}
$$

applies.

\section{b) Oblate Ellipsoids}

The major and minor semiaxes will again be denoted by $a$ and $b(a>b)$, hence the eccentricity is given by the same expression as in case a). The unit vector $\boldsymbol{u}$ characterizes the symmetry axis of the ellipsoid. The torque is given by Eq. (13), with

$$
\begin{gathered}
\gamma_{\|}=\alpha \gamma_{\mathrm{s}} g_{2}(\varepsilon), \\
\gamma_{\perp}=\frac{1}{2} \gamma_{\mathrm{s}}\left[\alpha\left(g_{2}(\varepsilon)+\left(1-\varepsilon^{2}\right) g_{1}(\varepsilon)\right)\right. \\
\left.+3\left(1-\frac{6-\pi}{8} \alpha\right) \varepsilon^{4} g_{3}(\varepsilon)\right] .
\end{gathered}
$$

The functions $g_{1}, g_{2}$ and $g_{3}$ are defined in the appendix. The two coefficients $\gamma_{\|}$and $\gamma_{\perp}$ are equal to $\alpha \gamma_{\mathrm{s}}$ for $\varepsilon=0$; for $\varepsilon=1$ the torque on a disk with radius $a$ is obtained which coincides with Equation (11). If the angular velocity lies in the plane of the disk, one gets

$$
\boldsymbol{M}=-\frac{1}{2} \varrho \bar{c} \pi a^{4}\left(1-\frac{4-\pi}{8} \alpha\right) \Omega,
$$

which is about twice as much as in Eq. (11). The ratio $\chi=\omega_{\|} / \omega_{\perp}$ is

$$
\begin{aligned}
\chi=\alpha\left(2-\varepsilon^{2}\right) g_{2} & {\left[\alpha\left(g_{2}+\left(1-\varepsilon^{2}\right) g_{1}\right)\right.} \\
+ & \left.3\left(1-\frac{6-\pi}{8} \alpha\right) \varepsilon^{4} g_{3}\right]^{-1} .
\end{aligned}
$$


The inequality

$$
1 \geqq \chi \geqq \alpha\left[\alpha+4\left(1-\frac{6-\pi}{8} \alpha\right)\right]^{-1}
$$

is valid for $\varepsilon$ in the range $0 \leqq \varepsilon \leqq 1$.

\section{Appendix}

The integrals occurring in Eq. (9) will be calculated for the two types of ellipsoids.
A) Prolate Ellipsoids

The surface of the ellipsoid is described by

$$
\begin{aligned}
& x=b \sin \psi \cos \varphi \\
& y=b \sin \psi \sin \varphi \\
& z=a \cos \psi .
\end{aligned}
$$

Each point of the surface is characterized by two angles $\varphi$ and $\psi(0 \leqq \varphi \leqq 2 \pi, 0 \leqq \psi \leqq \pi)$. The symmetry axis $\boldsymbol{u}$ is chosen as $z$-axis $\left(\boldsymbol{e}_{z}=\boldsymbol{u}\right)$. Since $a>b$ for the prolate ellipsoid, the eccentricity is

given by $\varepsilon=\left(1-b^{2} / a^{2}\right)^{1 / 2}$. The surface element $\mathrm{d} S$ and the unit vector $\boldsymbol{n}$ are

$$
\begin{gathered}
\mathrm{d} S=a^{2}\left(1-\varepsilon^{2}\right)^{1 / 2}\left(1-\varepsilon^{2} \cos ^{2} \psi\right)^{1 / 2} \sin \psi \mathrm{d} \psi \mathrm{d} \varphi \\
\left(1-\varepsilon^{2} \cos ^{2} \psi\right)^{1 / 2} \boldsymbol{n}=\sin \psi \cos \varphi \boldsymbol{e}_{x}+\sin \psi \sin \varphi \boldsymbol{e}_{y}+\left(1-\varepsilon^{2}\right)^{1 / 2} \cos \psi \boldsymbol{u},
\end{gathered}
$$

where $\boldsymbol{e}_{x}$ and $\boldsymbol{e}_{y}$ are unit vectors along the $x$ and $y$-axis. One needs the following relation

$$
a\left(1-\varepsilon^{2}\right)^{1 / 2}\left(1-\varepsilon^{2} \cos ^{2} \psi\right)^{1 / 2} \boldsymbol{x} \times \boldsymbol{n}=\varepsilon^{2} \boldsymbol{x} \cdot \boldsymbol{u}(\boldsymbol{u} \times \boldsymbol{x}),
$$

which is easily derived from Equation (A 1), (A 3). For the calculation of the torque it is sufficient to consider integrals of the form $\int x_{\mu} x_{\nu} \mathrm{d} S$ and $\int(\boldsymbol{x} \times \boldsymbol{n})_{\mu}(\boldsymbol{x} \times \boldsymbol{n})_{\nu} \mathrm{d} S$. By using Eqs. (A 1-A 4), they can be written as

$$
\begin{array}{r}
\int x_{\mu} x_{v} \mathrm{~d} S=\frac{4 \pi}{3} a^{4}\left(1-\varepsilon^{2}\right)^{1 / 2}\left[\left(1-\varepsilon^{2}\right) f_{2}\left(\delta_{\mu v}-u_{\mu} u_{v}\right)+f_{1} u_{\mu} u_{v}\right], \\
\int(\boldsymbol{x} \times \boldsymbol{n})_{\mu}(\boldsymbol{x} \times \boldsymbol{n})_{v} \mathrm{~d} S=\pi a^{4}\left(1-\varepsilon^{2}\right)^{1 / 2} \varepsilon^{4} f_{3}\left(\delta_{\mu v}-u_{\mu} u_{v}\right),
\end{array}
$$

where the functions $f_{1}, f_{2}$, and $f_{3}$ are defined by

$$
\begin{aligned}
& f_{1}(\varepsilon)=\frac{3}{2} \int_{0}^{\pi} \cos ^{2} \psi \sin \psi^{\prime}\left(1-\varepsilon^{2} \cos ^{2} \psi\right)^{1 / 2} \mathrm{~d} \psi, \\
& f_{2}(\varepsilon)=\frac{3}{4} \int_{0}^{\pi} \sin ^{3} \psi\left(1-\varepsilon^{2} \cos ^{2} \psi^{\prime}\right)^{1 / 2} \mathrm{~d} \psi, \\
& f_{3}(\varepsilon)=\int_{0}^{\pi} \sin ^{3} \psi \cos ^{2} \psi\left(1-\varepsilon^{2} \cos ^{2} \psi^{\prime}\right)^{-1 / 2} \mathrm{~d} \psi^{\prime} .
\end{aligned}
$$

By the substitution $\cos y^{\prime}=\xi$ they can be expressed by elementary funotions. The result is:

$$
\begin{aligned}
& f_{1}=\frac{3}{8} \varepsilon^{-2}\left[\frac{1}{\varepsilon} \arcsin \varepsilon-\left(1-2 \varepsilon^{2}\right)\left(1-\varepsilon^{2}\right)^{1 / 2}\right], \\
& f_{2}=\frac{3}{16} \varepsilon^{-2}\left[\left(1+2 \varepsilon^{2}\right)\left(1-\varepsilon^{2}\right)^{1 / 2}-\left(1-4 \varepsilon^{2}\right) \frac{1}{\varepsilon} \arcsin \varepsilon\right], \\
& f_{3}=\frac{1}{4} \varepsilon^{-4}\left[\left(3-2 \varepsilon^{2}\right)\left(1-\varepsilon^{2}\right)^{1 / 2}+\left(4 \varepsilon^{2}-3\right) \frac{1}{\varepsilon} \arcsin \varepsilon\right] .
\end{aligned}
$$

Some special values of interest are:

$$
f_{1}(0)=1, \quad f_{2}(0)=1, f_{3}(0)=4 / 15, f_{1}(1)=3 \pi / 16, f_{2}(1)=9 \pi / 32, f_{3}(1)=\pi / 8 .
$$

These values are used to calculate $\chi=\omega \| / \omega_{\perp}$ for $\varepsilon=0$ and $\varepsilon=1$. 


\section{B) Oblate Ellipsoids}

The surface of the ellipsoid is described by

$$
x=a \sin \psi \cos \varphi, \quad y=a \sin \psi \sin \varphi, \quad z=b \cos \psi .
$$

The unit vector $\boldsymbol{u}$ of the symmetry axis is along the positive $z$-direction. Since $a>b$, the eccentricity is given by the same expression as in case A). The surface element $\mathrm{d} S$ and the normal $\boldsymbol{n}$ are

$$
\begin{gathered}
\mathrm{d} S=a^{2}\left(1-\varepsilon^{2} \sin ^{2} \psi\right)^{1 / 2} \sin \psi \mathrm{d} \psi \mathrm{d} \varphi, \\
\left(1-\varepsilon^{2} \sin ^{2} \psi\right)^{1 / 2} \boldsymbol{n}=\left(1-\varepsilon^{2}\right)^{1 / 2}\left(\sin \psi \cos \varphi \boldsymbol{e}_{x}+\sin \psi \sin \varphi \boldsymbol{e}_{y}\right)+\cos \psi \boldsymbol{u} .
\end{gathered}
$$

The relation corresponding to Eq. (A 4) is

$$
a\left(1-\varepsilon^{2}\right)^{1 / 2}\left(1-\varepsilon^{2} \sin ^{2} \psi\right)^{1 / 2} \boldsymbol{x} \times \boldsymbol{n}=\varepsilon^{2} \boldsymbol{x} \cdot \boldsymbol{u}(\boldsymbol{x} \times \boldsymbol{u}) .
$$

The two integrals of interest are

$$
\begin{aligned}
& \int x_{\mu} x_{v} \mathrm{~d} S=\frac{4 \pi}{3} a^{4}\left[g_{2}\left(\delta_{\mu v}-u_{\mu} u_{v}\right)+\left(1-\varepsilon^{2}\right) g_{1} u_{\mu} u_{v}\right], \\
& \int(\boldsymbol{x} \times \boldsymbol{n})_{\mu}(\boldsymbol{x} \times \boldsymbol{n})_{v} \mathrm{~d} S=\pi a^{4} \varepsilon^{4} g_{3}\left(\delta_{\mu v}-u_{\mu} u_{v}\right),
\end{aligned}
$$

where the functions $g_{1}, g_{2}$, and $g_{3}$ are defined by

$$
\begin{aligned}
& g_{1}(\varepsilon)=3 / 2 \int_{0}^{\pi} \cos ^{2} \psi \sin \psi\left(1-\varepsilon^{2} \sin ^{2} \psi\right)^{1 / 2} \mathrm{~d} \psi, \\
& g_{2}(\varepsilon)=3 / 4 \int_{0}^{\pi} \sin ^{3} \psi\left(1-\varepsilon^{2} \sin ^{2} \psi\right)^{1 / 2} \mathrm{~d} \psi, \\
& g_{3}(\varepsilon)=\int_{0}^{\pi} \sin ^{3} \psi \cos ^{2} \psi\left(1-\varepsilon^{2} \sin ^{2} \psi\right)^{-1 / 2} \mathrm{~d} \psi .
\end{aligned}
$$

Using the same substitution $\cos \psi=\xi$ as before, these integrals can easily be calculated. One obtains:

$$
\begin{aligned}
& g_{1}=3 / 8 \varepsilon^{-2}\left[\left(1+\varepsilon^{2}\right)-\frac{\left(1-\varepsilon^{2}\right)^{2}}{2 \varepsilon} \ln \left(\frac{1+\varepsilon}{1-\varepsilon}\right)\right], \\
& g_{2}=3 / 16 \varepsilon^{-2}\left[\left(3 \varepsilon^{2}-1\right)+\left(3 \varepsilon^{2}+1\right) \frac{\left(1-\varepsilon^{2}\right)}{2 \varepsilon} \ln \left(\frac{1+\varepsilon}{1-\varepsilon}\right)\right], \\
& g_{3}=1 / 4 \varepsilon^{-4}\left[\left(3-\varepsilon^{2}\right)-\left(3+\varepsilon^{2}\right) \frac{\left(1-\varepsilon^{2}\right)}{2 \varepsilon} \ln \left(\frac{1+\varepsilon}{1-\varepsilon}\right)\right] .
\end{aligned}
$$

Some important values are:

$$
\begin{aligned}
& g_{1}(0)=1, \quad g_{2}(0)=1, g_{3}(0)=4 / 15, \\
& g_{1}(1)=3 / 4, g_{2}(1)=3 / 8, g_{3}(1)=1 / 2 \text {. }
\end{aligned}
$$

It should be noticed that Eq. (B 1) is obtained from Eq. (A 1 ) by interchanging $a$ and $b$. This is equivalent to replacing $\varepsilon$ by

$$
\varepsilon^{\prime}=i \varepsilon\left(1-\varepsilon^{2}\right)^{-1 / s} \text {. }
$$

As is to be seen from Eqs. (A 7 - A 9) and Eqs. (B 7 to B 9), the functions $g_{1}, g_{2}$, and $g_{3}$ are connected with $f_{1}, f_{2}$, and $f_{3}$ in the following way:

$$
\begin{aligned}
& g_{1}(\varepsilon)=\left(1-\varepsilon^{2}\right)^{1 / 2} f_{1}\left(\varepsilon^{\prime}\right), \\
& g_{2}(\varepsilon)=\left(1-\varepsilon^{2}\right)^{1 / 2} f_{2}\left(\varepsilon^{\prime}\right), \\
& g_{3}(\varepsilon)=\left(1-\varepsilon^{2}\right)^{-1 / 2} f_{3}\left(\varepsilon^{\prime}\right) .
\end{aligned}
$$

Use has been made of the relation

$$
\arcsin \left[i \varepsilon\left(1-\varepsilon^{2}\right)^{-1 / 2}\right]=\frac{i}{2} \ln \left(\frac{1+\varepsilon}{1-\varepsilon}\right) .
$$

Finally, Eqs. (18 a, $18 \mathrm{~b}$ ) can be obtained from Eqs. $(14 \mathrm{a}, 14 \mathrm{~b})$ if in addition $\gamma_{\mathrm{s}}$ is replaced by $\left(1-\varepsilon^{2}\right)^{2} \gamma_{s}$. 


\section{Acknowledgement}

The author thanks Prof. L. Waldmann, Dr. S. Hess, and Dr. W. E. Köhler for discussions.

1 P. S. Epstein, Phys. Rev. 23, 710 (1924).

2 R. Gans, Ann. Physik 86, 628 [1928].
3 H. Lamb, Hydrodynamics, University Press, Cambridge 1932, p. 139.

${ }^{4}$ L. Waldmann, Z. Naturforsch. 14 a, 589 [1959]. 